\title{
Inhibiting aberrant p53-PUMA feedback loop activation attenuates ischaemia reperfusion-induced neuroapoptosis and neuroinflammation in rats by downregulating caspase 3 and the NF-KB cytokine pathway
}

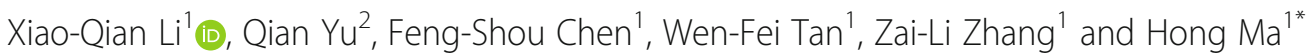

\begin{abstract}
Background: Ischaemia reperfusion (IR) induces multiple pathophysiological changes. In addition to its classical role in regulating tumourigenesis, the feedback loop formed by p53 and its driven target p53-upregulated modulator of apoptosis (PUMA) was recently demonstrated to be the common node tightly controlling various cellular responses during myocardial IR. However, the roles of the p53-PUMA feedback loop in the spinal cord remain unclear. This study aimed to elucidate the roles of p53-PUMA feedback interactions in the spinal cord after IR, specifically investigating their regulation of caspase 3-mediated apoptosis and nuclear factor (NF)-kB-mediated cytokine release.

Methods: SD rats subjected to 12 min of aortic arch occlusion served as IR models. Neurological assessment as well as p53 and PUMA mRNA and protein expression analyses were performed at 12-h intervals during a 48-h reperfusion period. The cellular distributions of p53 and PUMA were determined via double immunofluorescence staining. The effects of the p53-PUMA feedback loop on modulating hind-limb function; the number of TUNELpositive cells; and protein levels of caspase 3, NF-kB and cytokines interleukin (IL)-1 $\beta$ and tumour necrosis factor (TNF)- $a$, were evaluated by intrathecal treatment with PUMA-specific or scramble siRNA and pifithrin (PFT)-a. Bloodspinal cord barrier (BSCB) breakdown was examined by Evans blue (EB) extravasation and water content analyses.

Results: IR induced significant behavioural deficits as demonstrated by deceased Tarlov scores, which displayed trends opposite those of PUMA and p53 protein and mRNA expression. Upregulated PUMA and p53 fluorescent labels were widely distributed in neurons, astrocytes and microglia. Injecting si-PUMA and PFT-a exerted significant anti-apoptosis effects as shown by the reduced number of TUNEL-positive cells, nuclear abnormalities and cleaved caspase 3 levels at $48 \mathrm{~h}$ post-IR. Additionally, p53 colocalized with NF-KB within the cell. Similarly, injecting si-PUMA and PFT-a exerted anti-inflammatory effects as shown by the decreased NF-KB translocation and release of IL-1 $\beta$ and TNF-a. Additionally, injecting si-PUMA and PFT-a preserved the BSCB integrity as determined by decreased EB extravasation and spinal water content. However, injecting si-Con did not induce any of the abovementioned effects.

(Continued on next page)
\end{abstract}

\footnotetext{
* Correspondence: mahong5466@yahoo.com

'Department of Anesthesiology, First Affiliated Hospital, China Medical

University, Shenyang 110001, Liaoning, China

Full list of author information is available at the end of the article
}

(c) The Author(s). 2018 Open Access This article is distributed under the terms of the Creative Commons Attribution 4.0 International License (http://creativecommons.org/licenses/by/4.0/), which permits unrestricted use, distribution, and reproduction in any medium, provided you give appropriate credit to the original author(s) and the source, provide a link to the Creative Commons license, and indicate if changes were made. The Creative Commons Public Domain Dedication waiver (http://creativecommons.org/publicdomain/zero/1.0/) applies to the data made available in this article, unless otherwise stated. 
(Continued from previous page)

Conclusions: Inhibition of aberrant p53-PUMA feedback loop activation by intrathecal treatment with si-PUMA and PFT-a prevented IR-induced neuroapoptosis, inflammatory responses and BSCB breakdown by inactivating caspase 3-mediated apoptosis and NF-kB-mediated cytokine release.

Keywords: Apoptosis, Blood-spinal cord barrier, Inflammation, Ischaemia reperfusion, p53, p53 upregulated modulator of apoptosis

\section{Background}

Spinal cord ischemia reperfusion (IR) injury is a major challenge and severe complication during cardiothoracic and vascular surgery [1], as it definitively induces excessive and extensive secondary injuries due to a wide range of pathological changes. Spinal cord IR injury elicits a concomitant increase in blood-spinal cord barrier (BSCB) permeability, accompanied by the subsequent activation of proinflammatory responses and cellular programmed or autophagyassociated death [2, 3]. Commonly, these responses are regulated by separate signalling pathways, and recent emerging evidence indicates that they may coordinate and integrate into a signalling network in response to ischaemia $[4,5]$. As a stress sensor under stimuli, the tumour suppressor transcription factor p53 drives the expression of multiple targets to execute p53-mediated cellular functions [6-8]. In addition to its classical role in regulating tumour metastasis, p53 was newly demonstrated to be a common node in a signalling network that initiates apoptosis and inflammation during brain ischaemia $[9,10]$. Upon ischaemia, p53 rapidly accumulates in the injured zone and tightly controls the balance between pro-apoptotic proteins (e.g., BH3interacting domain death agonist, Bid) and anti-apoptotic proteins (e.g., B-cell lymphoma 2, Bcl-2) by promoting a feedback interaction with the murine double minute 2 (MDM2) gene [7, 11]. Moreover, p53 is thought to regulate not only the p53-mediated pathway but also other pathways that participate in various diseases models $[6,12,13]$. Pharmacologically blocking p53 function by pifithrin (PFT)- $\alpha$ induced significant neuroprotective effects by inhibiting nuclear factor (NF)- $\mathrm{kB}$ transcription and releasing the downstream proinflammatory cytokines interleukin (IL)- $1 \beta$ and tumour necrosis factor (TNF)- $\alpha$ [14]. In addition to MDM2, p53 upregulated modulator of apoptosis (PUMA) has gradually received substantial attention among a list of p53-driven targets $[8,13,15]$. A BH3-only protein (BH3) in the $\mathrm{Bcl}-2$ protein family, PUMA is expressed at a very low level under normal conditions. PUMA can be induced immediately by upregulation of the p53 level and acts as a crucial checkpoint that finely controls further p53-dependent network activation in different models of diseases $[8,15,16]$. Therefore, regulating the levels and function of PUMA is expected to become a new therapeutic target against IR [13]. In agreement with this hypothesis, silencing PUMA expression using knockout mice or the targeting RNA interference technique significantly decreased its ability to interact with p53 during ischaemic injury, which further prevented against p53 transactivation and cellular apoptosis $[15,17]$. Thus, better understanding on how to properly regulate the p53-PUMA feedback loop in vivo may significantly impact the development of new therapies. However, little is known about a common node of p53 and how the p53-PUMA feedback loop functions via both apoptotic and proinflammatory mechanisms during spinal cord IR. In this context, we first elucidated the IR-induced temporal profiles of p53 and PUMA expressed in the spinal cord to investigate the potential interactions in an adult rat model of IR. We then assessed the effects of the p53-PUMA feedback loop on hind-limb motor function; the number of TUNEL-positive cells; and the protein levels of caspase 3, NF- $-\mathrm{B}$, cytokines IL- $1 \beta$, and TNF- $\alpha$, in vivo by the intrathecal injection of specific PUMA siRNA (si-PUMA), scramble siRNA (si-Con) and PFT- $\alpha$, a p53-specific inhibitor. Additionally, the effects of si-PUMA and PFT- $\alpha$ on preventing BSCB leakage were evaluated to further determine the inflammatory responses after IR.

\section{Methods \\ Animals}

Eight-week-old Sprague Dawley (SD) rats, weighing approximately $250 \mathrm{~g}$, were purchased from the Animal Center of China Medical University (Shenyang, China). The rats had free access to food and water and housed in a standard cage that was maintained at $22-24{ }^{\circ} \mathrm{C}$, relative humidity of $50-60 \%$ with a $12 / 12$-h light/dark cycle for 1 week before surgery.

\section{Rat model of spinal cord IR injury}

The rat IR model was established as we previously reported [18]. After anaesthetization via an intraperitoneal injection of $10 \%$ chloral hydrate $(300 \mathrm{mg} / \mathrm{kg}$ body weight; Beyotime Biotechnology, Shanghai, China), the rats were mechanically ventilated by endotracheal intubation. Two 24-gauge catheters were separately inserted into the left carotid artery or the tail artery to measure the proximal and distal blood pressure, respectively. By directly envisioning the left thoracotomy, the aortic arch was exposed and clamped just distal to the left subclavian artery to induce ischaemia, which was confirmed by the observation of a distal blood pressure less than 
$10 \mathrm{mmHg}$. After $12 \mathrm{~min}$, the clamp was removed to initiate the $48 \mathrm{~h}$ reperfusion period. Rats in the sham group were subjected to the same surgery without clamping.

\section{Intrathecal injection of PUMA siRNA and control}

The rat sequences of si-PUMA and si-Con were constructed by Genechem (Shanghai, China) according to effective interference sites previously reported [16]. The sequences of si-PUMA and si-Con are as follows: si-PUMA, 5 '-CGUG UGACCACUGGCAUUC-3' and 5'-GAAUGCCAGUGGU CACACG-3; and si-Con, 5'-UUCUCCGAACGUGUCACG U-3' and 5' ${ }^{\prime}$-ACGUGACACGUUCGGAGAA-3'. si-PUMA and si-Con were transfected in vivo via intrathecal injection together with Lipofectamine 3000 (Invitrogen, CA, USA) as reported previously [18]. The injection position was located at the $\mathrm{L}_{5-6}$ segments of the dura. Each injection was administered at the same time of day, and the correct position was confirmed by the tail flick phenomenon [18]. After three consecutive injections, PUMA mRNA expression levels were detected to determine the optimal interference concentration from four adjacent concentration gradients (50, 100, 200 and $300 \mu \mathrm{mol} / \mathrm{L}$ ). Based on the above, $100 \mu \mathrm{mol} / \mathrm{L}$ was selected as the final concentration because it was the lowest concentration that significantly downregulated PUMA mRNA expression. In addition, only rats with normal motor function after three injections were included in this study.

\section{Experimental protocol}

The rats were randomly assigned into groups $(n=6$ per group) as shown in Fig. 1. The sham group rats were subjected to the surgical procedure without ischaemia, and rats in all other groups underwent ischaemia after three consecutive intrathecal injections. Each injection contained $15 \mu \mathrm{L}$ of total fluid comprising $2 \%$ dimethyl sulfoxide (DMSO), si-PUMA, si-Con, or PFT- $\alpha$ (a p53 inhibitor, $1 \mu \mathrm{g} / \mu \mathrm{L}$, Selleck Chemicals, S2929, PA, USA). Rats were euthanized at $48 \mathrm{~h}$ after surgery with an overdose of sevoflurane. Spinal cord segments $\mathrm{L}_{4-6}$ were collected for further analysis.

\section{Neurological assessment}

For $48 \mathrm{~h}$ post-IR, hind-limb neurological functions were detected at 12-h intervals using modified Tarlov criteria. The functions were quantified by ambulation and ranged from 0 (no ankle movement) to 4 (normal). The assessments were performed by two observers who were blinded to the entire experimental protocol [19].

\section{Quantification of $\mathrm{p} 53$ and PUMA mRNA expression}

Total RNAs from dissected $\mathrm{L}_{4-6}$ spinal cord segments were extracted using TRIzol reagent (Beyotime, Beijing, China). First-strand cDNAs were transcribed using the PrimeScript RT Reagent Kit (Takara, Tokyo, Japan), incubated with the corresponding primers and SYBR Green SuperMix-UDG (Thermo Fisher Scientific, MA, USA), and analysed on a Prism 7000 detection system (Applied Biosystems, CA, USA). Each gene was quantified in triplicate, and the levels were normalized to those of $\beta$-actin. Data were analysed using the $2^{-\Delta \Delta C t}$ method. The following primers were used: p53 (forward, 5'-CCCAGGGAG TGCAAAGAGAG-3' and reverse, 5'-TCTCGGAACA TCTCGAAGCG-3'); PUMA (forward, 5' -GTGTGGAGG AGGAGGAGTGG-3' and reverse, $5^{\prime}$-TCGGTGTCGAT GTTGCTCTT-3'); and $\beta$-actin (forward, 5'-CAGGAG ATGGCCACTGCCGCA-3', and reverse, $5^{\prime}$-TCCTTCT GCATCCTGTCAGCAC-3').

\section{Double immunofluorescence staining}

The cellular distribution of PUMA and p53 expression after IR was determined by double immunofluorescence staining at $48 \mathrm{~h}$ post-IR, as the lowest behavioural scores were also measured at that time point [18]. Briefly, the spinal cord tissues were frozen and sliced into $20-\mu \mathrm{m}$-thick sections. After permeabilization and blocking with $10 \%$ bovine

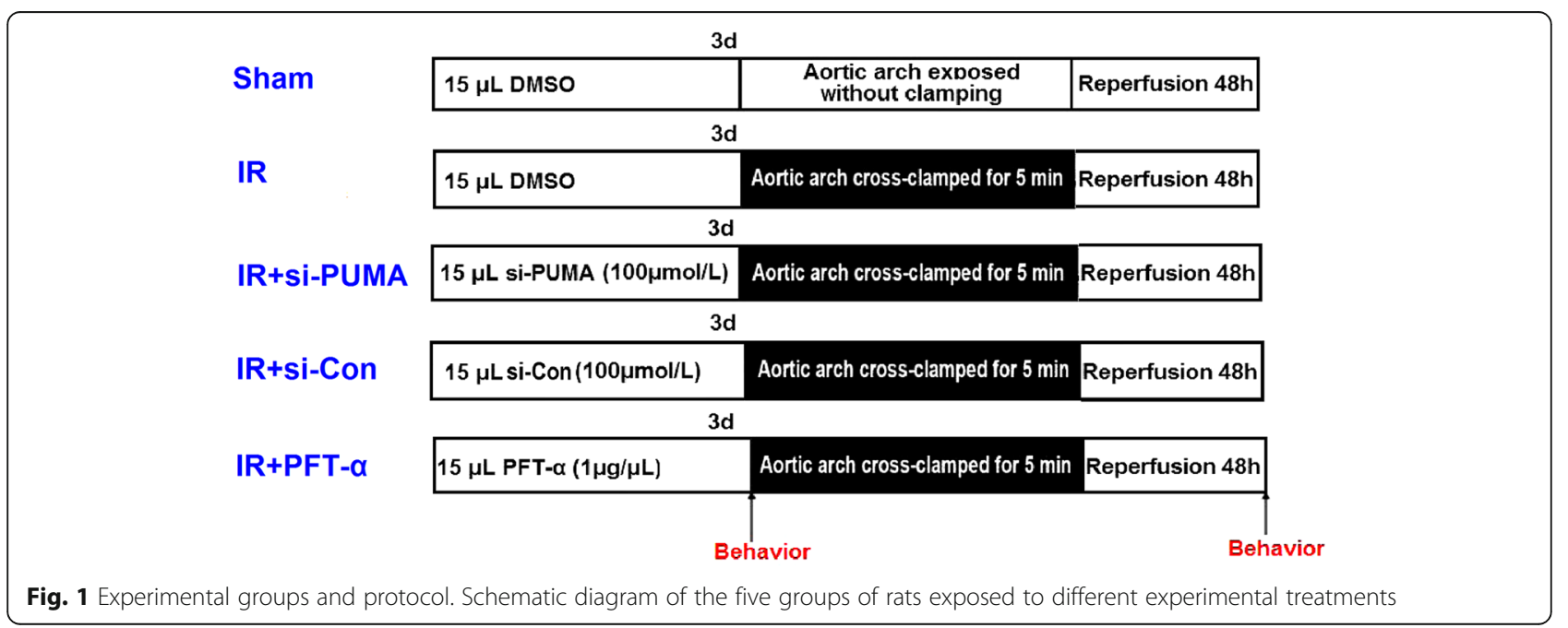


serum albumin (BSA), the slices were incubated overnight at $4{ }^{\circ} \mathrm{C}$ with the rabbit anti-PUMA (Abcam, ab9643, 1:100, MA, USA) or rabbit anti-p53 primary antibody (Abcam, ab131442, 1:200) and specific cellular marker primary antibodies as follows: mouse anti-NeuN (neuronal marker, Abcam, ab104224, 1:500), mouse anti-GFAP (astrocytic marker, Abcam, ab10062,1:500) and mouse anti-Iba-1 (microglial marker, Abcam, ab15690,1:400). The samples were then incubated with the secondary reagents Alexa 488-conjugated donkey anti-mouse IgG (1:500, Life Technologies, CA, USA) and Alexa 594-conjugated donkey anti-rabbit IgG (1:500, Life Technologies) for $2 \mathrm{~h}$ at room temperature to visualize the staining. Nonspecific staining was excluded by incubation with nonimmune serum but no primary antibody. Representative images of anteriorl horns were captured at $400 \times$ magnification on the Leica TCS SP2 fluorescence microscope (Leica Microsystems, Buffalo Grove, IL, USA). Quantified fluorescence intensities and numbers of immunoreactive cells were averaged for three stained slices per antibody.

Additionally, potential interactions among p53, PUMA and NF- $\mathrm{kB}$ p65 were also detected exactly as described above with the following primary antibodies: mouse anti-p53 (Abcam, ab26, 1:200), rabbit anti-PUMA (Abcam, ab9643, 1:100), rabbit anti-NF-kB (Abcam, ab16502, 1:100) and mouse anti-NF-kB (Cell Signalling Technology, no. 6956, MA, USA).

\section{Western blotting}

$\mathrm{L}_{4-6}$ spinal cord segments were rapidly collected and homogenized on ice due to their vulnerability to ischaemic injury [20]. The cytosolic and nuclear fractions were extracted and purified with a nuclear and cytosol protein extraction kit (KangChen, KC415, Shanghai, China) [18]. The mitochondrial fractionation was performed according to a multiple centrifugation method described previously [15]. Briefly, the homogenates were first centrifuged at $750 \mathrm{~g}$ at $4{ }^{\circ} \mathrm{C}$ and then at $8000 \mathrm{~g}$ for $20 \mathrm{~min}$ at $4{ }^{\circ} \mathrm{C}$. The pellets obtained were considered the mitochondrial fraction. The samples were separated by gel electrophoresis and then transferred to polyvinylidene difluoride membranes. After blocking with $5 \%$ non-fat milk, the membranes were incubated overnight at $4{ }^{\circ} \mathrm{C}$ with primary antibodies against p53 (Abcam, ab26, 1:200), PUMA (Abcam, ab9643, 1:200), cleaved caspase 3 (Cell Signaling Technology, \#9661, 1:300) and NF-kB p65 (phospho S536, Abcam, ab86299, 1:100). $\beta$-actin (Abcam, ab8227, 1:10000), histone (Abcam, ab10799, 1:5000) and COX-IV (Abcam, ab33985, 1:5000) were used as the controls. The bands were visualized by an enhanced chemiluminescence (ECL) kit (Beyotime, Beijing, China) and quantified using Quantity One software (Bio-Rad Laboratories, Milan, Italy).

\section{Terminal deoxynucleotidyl transferase-mediated dUTP} nick-end labelling (TUNEL) assay

The TUNEL assay was performed to identify cellular apoptosis as previously described [3]. After fixation in 4\% formaldehyde, the spinal sections were stained with an ApopTag Fluorescein In Situ Apoptosis Detection Kit (EMD Millipore, S7110) according to the manufacturer's instructions. Briefly, the sections were incubated with TUNEL reaction mixture and kept in the dark for $60 \mathrm{~min}$ at room temperature. Then, 4',6-diamidino-2-phenylindole (DAPI, Beyotime) was added for $5 \mathrm{~min}$ to label the cell nuclei. Nonspecific staining was determined by omitting the TdT enzyme from the reaction mixture. The average numbers of TUNEL-positive cell in the anterior regions of three spinal sections were counted for comparison among the groups. Samples from each step were washed three times in phosphate buffered saline (PBS).

\section{Morphological analysis of apoptotic nuclei}

The morphology of apoptotic nuclei was visualized by DAPI staining [15]. Briefly, after fixation in $4 \%$ paraformaldehyde, the sections were stained with DAPI solution for $2 \mathrm{~min}$ at room temperature. Representative images of the anterior horns were captured at $400 \times$ magnification, and the number of nuclear abnormalities was averaged in three randomly selected slices.

\section{BSCB permeability assessment}

Evans blue (EB) extravasation and spinal water content analyses were performed to detect the integrity and permeability of BSCB as previously described [18]. Briefly, $2 \%$ EB ( $35 \mathrm{mg} / \mathrm{kg}$, Sigma, MO, USA) was slowly administered via the tail vein $30 \mathrm{~min}$ before euthanization. After EB circulated for $20-30 \mathrm{~min}$, the rats were transcardially perfused with $180 \mathrm{~mL}$ of sterile saline at $60 \mathrm{~mL} / \mathrm{min}$. To detect fluorescence, the $\mathrm{L}_{4-6}$ segments were cut into $15-\mu \mathrm{m}$ sections and visualized under a BX-60 fluorescence microscope (Olympus, NY, USA) with a green filter. For quantitation, the tissues were incubated in $400 \mu \mathrm{L}$ of $\mathrm{N}, \mathrm{N}^{\prime}$-dimethylformamide at $50{ }^{\circ} \mathrm{C}$ for $72 \mathrm{~h}$. After centrifugation, the supernatants were collected and colorimetrically measured at $632 \mathrm{~nm}$. Concentrations were reported as the amount of EB per spinal cord wet tissue weight (microgramme per gram) according to a standard curve.

\section{Spinal cord Oedema assessment}

The water content was measured by wet-dry method to quantify the spinal cord oedema. Fresh tissues from non-perfused rats were immediately collected. Approximately $1 \mathrm{~cm}$ of the $\mathrm{L}_{4-6}$ segments of the spinal cords was weighed as wet weight. Then, the segments were dried at $110{ }^{\circ} \mathrm{C}$ for $24 \mathrm{~h}$ and reweighed as dry weight (Havppvel method). The percentage of water content 
was calculated using the following formula: spinal cord water content $(\%)=($ wet weight - dry weight $) /$ wet weight $\times 100 \%[21]$.

\section{Measurement of IL-1 $\beta$ and TNF- $\alpha$ by ELISA}

The spinal cords were collected, homogenized and centrifuged, and their IL- $1 \beta$ and TNF- $\alpha$ contents were measured with ELISA kits (R\&D Systems, MN, USA) according to the manufacturer's instructions [18]. Absorbance was detected at $450 \mathrm{~nm}$, and the content of each sample was based on the standard curve and expressed as picogram per milligram protein.

\section{Statistical analysis}

All statistical data were normally distributed and presented as the mean \pm SEM in this study. When compared between groups, the data were analysed with the $t$ test, one-way or two-way ANOVA followed by post hoc Tukey test by SPSS software (version 17.0, SPSS Inc., Chicago, USA). A $P<0.05$ was considered statistically significant.

\section{Results}

Temporal induction of p53, PUMA levels and behavioural deficits post-IR

Given that p53 and PUMA are required for IR-induced cardiomyocyte apoptosis in vitro [16], we hypothesized that they might partially function in spinal cords in vivo. We detected temporal changes in p53 and PUMA protein expression at 12-h intervals for $48 \mathrm{~h}$ post-IR. Compared with those in the sham group, the levels of p53 and PUMA in the reperfusion group significantly increased with time during the $48 \mathrm{~h}$ reperfusion period and peaked at $48 \mathrm{~h}$ (Fig. $2 \mathrm{a}-\mathrm{c}, P<0.05$ ), suggesting that potential feedback interactions occur during IR.

As expected, during the 48-h reperfusion period, temporal assessments of motor function according to Tarlov scores showed tendencies opposite those shown by the p53 and PUMA levels at all the observed time points. The average Tarlov scores in the IR group were much lower than those in the baseline and sham groups, with the lowest levels being observed at $48 \mathrm{~h}$ (Fig. $2 \mathrm{~d}, P<0.05$ ). Similarly, the individual scores for each rat shown in Fig. 2e confirmed the development of neurological dysfunctions after IR $(P<0.05)$. In addition, this coincidence indicated the involvement of p53-PUMA in IR-induced behavioural deficits.

\section{Cellular distribution of IR-induced p53 and PUMA expression post-IR}

To investigate the specific cellular changes in p53 and PUMA expression after IR, double immunofluorescence staining was performed at $48 \mathrm{~h}$ post-IR, at which point the maximal levels of p53 and PUMA were detected during reperfusion (Fig. 2). As shown in Fig. 3a, c, colocalization
A

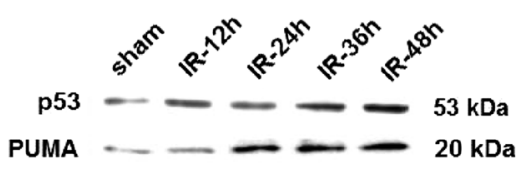

B

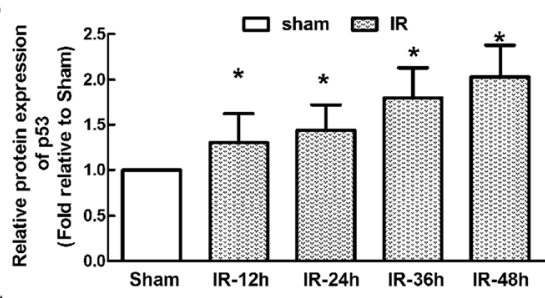

$\mathrm{C}$

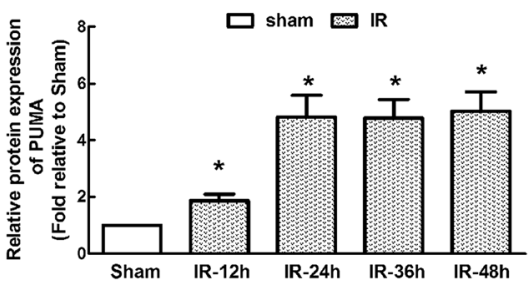

D

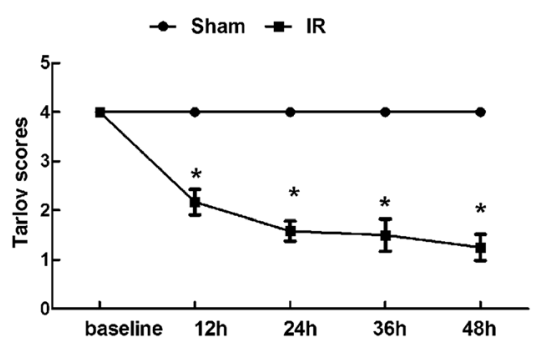

$\mathbf{E}$

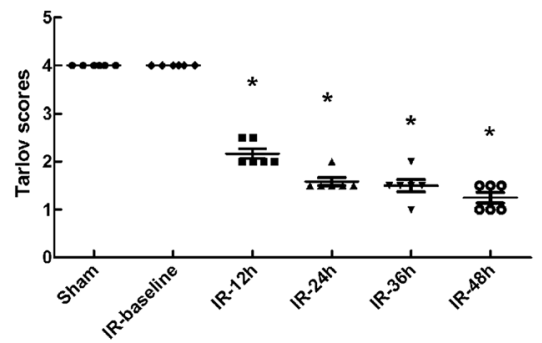

Fig. 2 Temporal profiles of IR-induced alteration in p53 and PUMA protein levels and neurological assessments. a Representative western blots of p53 and PUMA levels during the 48-h reperfusion period. $\mathbf{b}$ and $\mathbf{c}$ Quantification of the integrated intensities of p53 and PUMA bands. Data are expressed as the mean \pm SEM. The relative protein expression was normalised to that of the sham group. ${ }^{*} P<0.05$, versus the sham group. $\mathbf{d}$ Neurological assessments were scored using modified Tarlov criteria at 12-h intervals during $48 \mathrm{~h}$ of reperfusion. e Individual assessments of each rat at the indicated time points. Each symbol represents one mouse. The bar represents the median. Data are expressed as the mean \pm SEM. $n=6$ in each group. ${ }^{*} P<0.05$, versus the sham group 
A
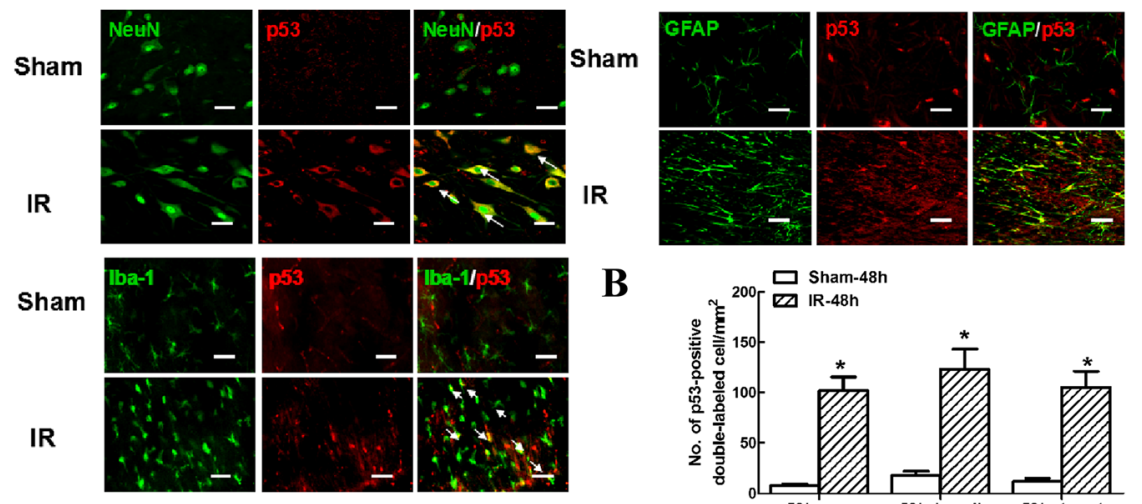

B

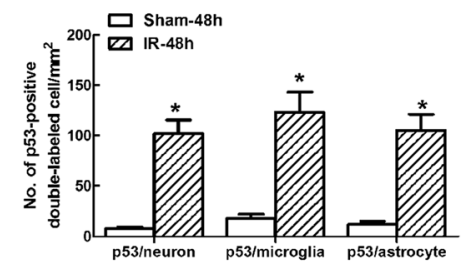

C
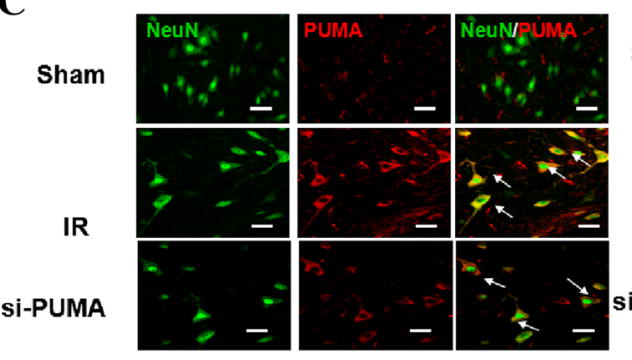

Sham
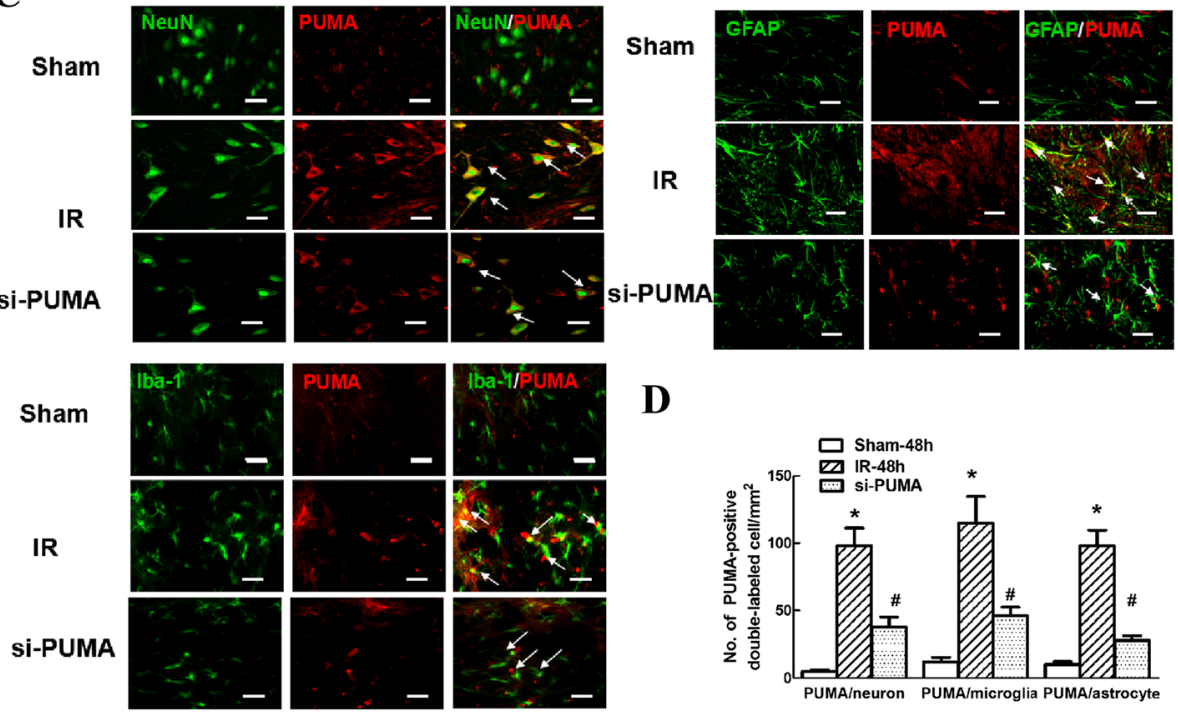

D

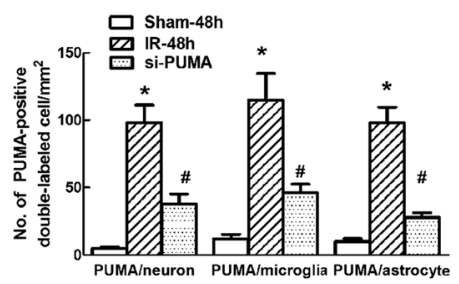

Fig. 3 Double immunofluorescence staining of p53 and PUMA with spinal major cellular markers after IR. a Representative immunofluorescence analysis of the colocalization of neurons (NeuN; green), microglia (Iba1; green) and astrocytes (GFAP; green) with p53 (red) in the anterior horns of grey matter at $48 \mathrm{~h}$ after IR. The arrows indicate co-localization with yellow labelling. Scale bars $=50 \mu \mathrm{m}$. b Quantification of the p53-positive neurons, astrocytes and microglia was performed and presented as the average of three independent images. Data are expressed as the mean \pm SEM. ${ }^{*} P<0.05$, versus the sham group. c Representative immunofluorescence analysis of the colocalization of neurons (NeuN; green), microglia (Iba1; green) and astrocytes (GFAP; green) with PUMA (red) in the grey matter of the anterior horn at $48 \mathrm{~h}$ after IR with or without si-PUMA treatment. Scale bars $=50 \mu \mathrm{m}$. d Quantification of PUMA-positive neurons, astrocytes and microglia was performed and presented as the average of three independent images. Data are expressed as the mean \pm SEM. ${ }^{*} P<0.05$, versus the sham group. ${ }^{*} P<0.05$, versus the $\mathbb{R}$ group

was displayed as a yellow fluorescent label under confocal microscopy. Compared with the sham group, significant increases in p53 and PUMA immunoreactivity were mainly distributed in cells positive for NeuN, GFAP and Iba-1 labelling, suggesting that p53 and PUMA were upregulated in both neurons and glial cells after IR. Similarly, quantitative analysis of the number of double-positive cells confirmed the above results (Fig. 3b, d, $P<0.05$ ).

Additionally, compared with IR group, intrathecal pretreatment with si-PUMA showed markedly lower PUMA immunoreactivity in all cell types of the spinal cord (Fig. 3c and d, $P<0.05$ ).
Intrathecal injection of si-PUMA and PFT-a prevented p53 and PUMA upregulation after IR

Because PUMA was shown to localize to mitochondria and induce cytochrome $\mathrm{c}$ release and subsequent caspase 3 activation [15], we detected PUMA levels in mitochondrial and cytosolic samples. As shown in representative western blots at $48 \mathrm{~h}$ post-IR, the intrathecal injection of si-PUMA and PFT- $\alpha$ significantly inhibited both the mitochondrial and cytosolic protein levels of PUMA, and these effects were not observed upon the injection of si-Con (Fig. 4a, $P<0.05$ ). No significant differences were observed between the IR and si-Con 


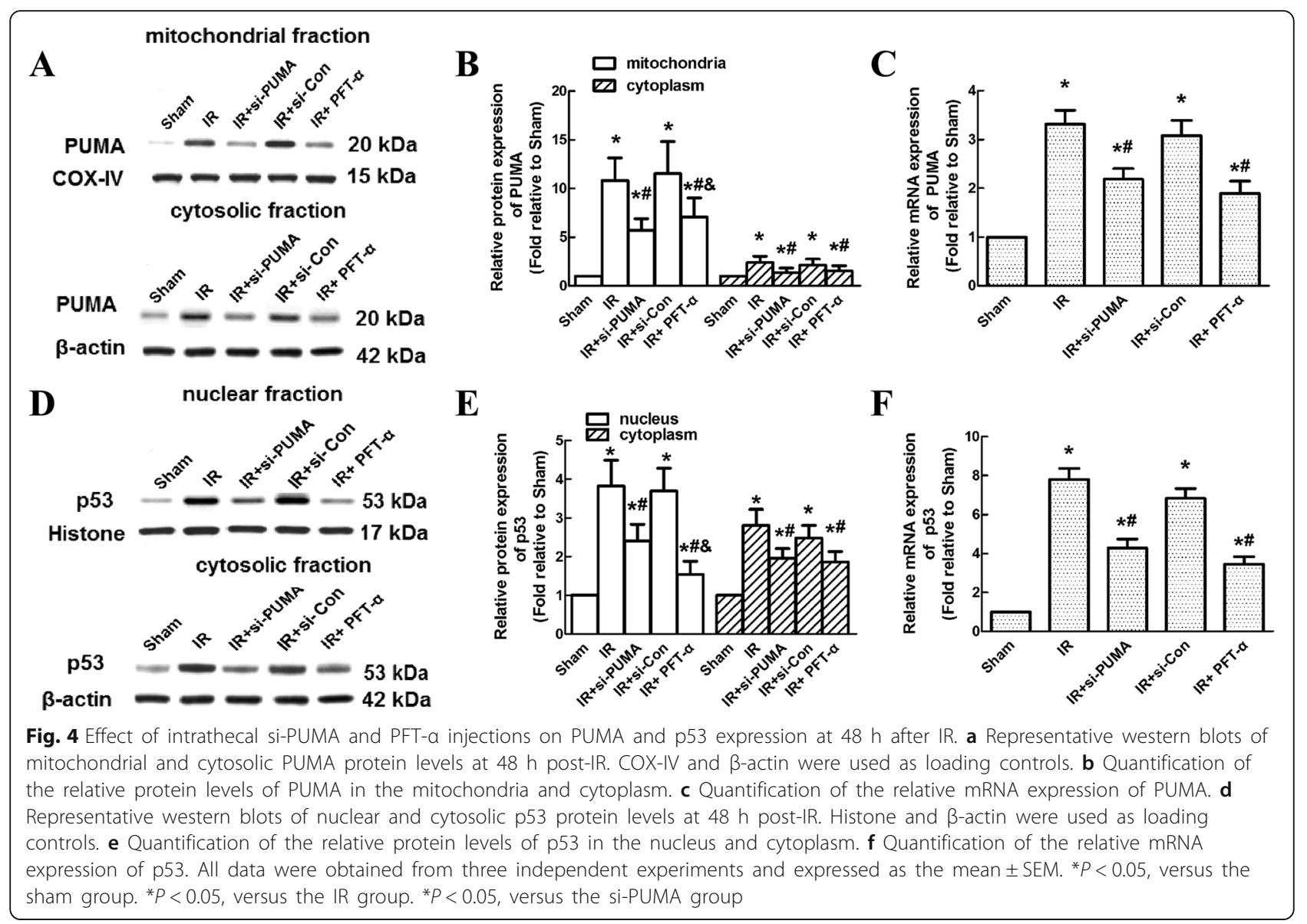

groups $(P>0.05)$. Quantitative analysis of PUMA protein and mRNA expression, shown in Fig. $4 \mathrm{~b}$ and c, confirmed the above results.

Given that p53 is the most important molecule upstream of PUMA and that the translocation of p53 from the cytoplasm to the nucleus signifies its activation, the protein levels of p53 in nuclear and cytoplasmic fractions were also determined. Compared with that in the IR group, the levels of p53 in both the nuclear and cytosolic samples were significantly decreased upon the intrathecal injection of si-PUMA and PFT- $\alpha$ (Fig. $4 \mathrm{~d}$ and e, $P<0.05$ ). Quantitative analysis of p53 protein and mRNA expression confirmed the above results (Fig. $4 \mathrm{e}$ and $\mathrm{f}, \mathrm{P}<0.05$ ). No significant differences were observed between the IR and si-Con groups $(P>0.05)$. Interestingly, lower protein levels of p53 and PUMA were observed in rats injected with PFT- $\alpha$ compared to those in rats injected with si-PUMA (Fig. 4a, b, d and e, $P<0.05$ ).

\section{Intrathecal injection of si-PUMA and PFT-a improved} behavioural function by inhibiting neuroapoptosis and downregulating caspase 3

Compared with those in sham-operated rats, all rats undergoing IR displayed hind-limb motor deficits, resulting in reduced Tarlov scores during the 48-h reperfusion period (Fig. 5a, $P<0.05$ ). Compared with those for untreated rats in the IR group, rats injected with si-PUMA and PFT- $\alpha$ had significantly higher average Tarlov scores $(P<0.05)$, whereas the rats injected with si-Con had comparable scores $(P>0.05)$. No significant differences were observed between the IR and si-Con groups $(P>0.05)$.

Based on previous studies [3, 22], cellular apoptosis is a common cause of behavioural impairment. Thus, we further assessed whether the PUMA-p53 loop helps regulates apoptosis using the TUNEL assay and measurement of cleaved caspase 3 levels. As shown in representative TUNEL staining, the green fluorescent spots indicated cellular apoptosis (Fig. 5b). Quantitative analysis showed significantly more TUNEL-positive cells in the IR injury groups than in the sham group (Fig. 5c, $P<0.05$ ). Compared with that in the IR group, increases in the number of TUNEL-positive cells in groups intrathecally injected with siRNA and PFT- $\alpha$ were significantly prevented $(P<0.05)$, whereas injection with si-Con did not induce these reductions $(P>0.05)$. These results were further confirmed by analysing the nucleic morphology by DAPI staining. As shown in Fig. 5d, normal nuclei were uniformly stained without condensation, and apoptotic nuclei displayed 


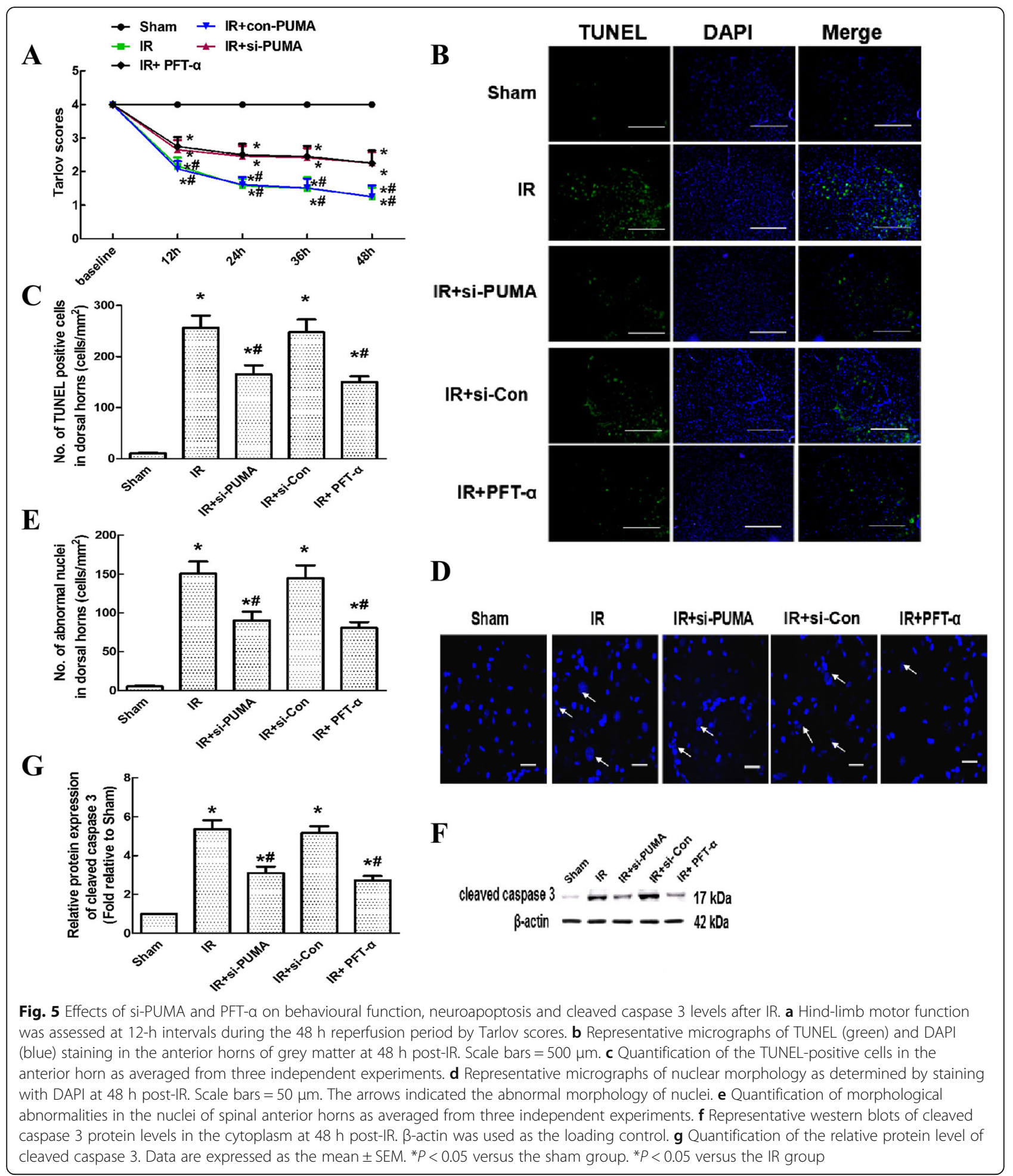

typical morphological features, such as abnormal nuclear size and nuclear fragmentation with some scattered apoptotic bodies. Similar to the TUNEL results, the number and morphology of nuclear abnormalities induced by IR were greatly ameliorated by the injection of siRNA and PFT- $\alpha$
(Fig. $5 \mathrm{~d}$ and e, $P<0.05$ ) but not by the injection of si-Con $(P>0.05)$.

Moreover, the levels of cleaved caspase 3, a well-known apoptotic marker, were detected by western blotting [22]. As shown in Fig. $5 \mathrm{f}$ and g, representative blots and quantitation 
showed that the patterns of cleaved caspase 3 protein levels among these groups were altered in a manner similar to those in the TUNEL assay $(P<0.05)$. No significant differences were observed between the IR and si-Con groups $(P>0.05)$.

\section{Intrathecal injection of si-PUMA and PFT-a inhibited neuroinflammation by inhibiting NF-KB-cytokine release after IR}

A previous chromatin immunoprecipitation assay showed that p53 and NF-kB p65 form a complex on the p53responsive MDM2 promoter, and further activation of NF- $\mathrm{kB}$ is linked to p53 phosphorylation [23]. Thus, the role of the PUMA-p53 loop in regulating neuroinflammation after spinal cord IR was hypothesized to involve NF-kB activation. As shown by double immunofluorescence staining, the fluorescent p53 labels were consistently distributed with the NF-kB labels (Fig. 6a). Compared with those in the sham group, the immunoreactivities of p53 and NF- $\mathrm{kB}$ were much stronger in the IR group (Fig. $6 \mathrm{~b}$ and $\mathrm{c}, P<0.05$ ). In addition, injection of si-PUMA and PFT- $\alpha$ significantly decreased both p53 and NF- $k B$ immunoreactivity as well as the number of p53/NF-kB double-positive cells (Fig. 6b-d, $P<0.05$ ), whereas no such changes were observed in the si-Con group $(P>0.05)$. Representative western blots further confirmed the above results. The nuclear localization and cytosolic phosphorylation of the p65 sub-unit have been used to indicate NF-kB activation [24]. Quantitative analysis showed that compared with those in the IR group, both the nuclear protein levels and cytosolic phosphorylation levels of NF- $\mathrm{kB}$ were significantly inhibited in the groups injected with si-PUMA and PFT- $\alpha$ (Fig. 6e and $f, P<0.05$ ), whereas no significant differences were observed in the si-Con group $(P>0.05)$. Similarly, downstream products of the NF-kB pathway were assessed by measuring the IL- $1 \beta$ and TNF- $\alpha$ concentrations. Consistently, alterations in the IL-1 $\beta$ and TNF- $\alpha$ concentrations were similar to those in NF-kB protein levels (Fig. $6 \mathrm{~g}$ and $\mathrm{h}, P<0.05$ ).

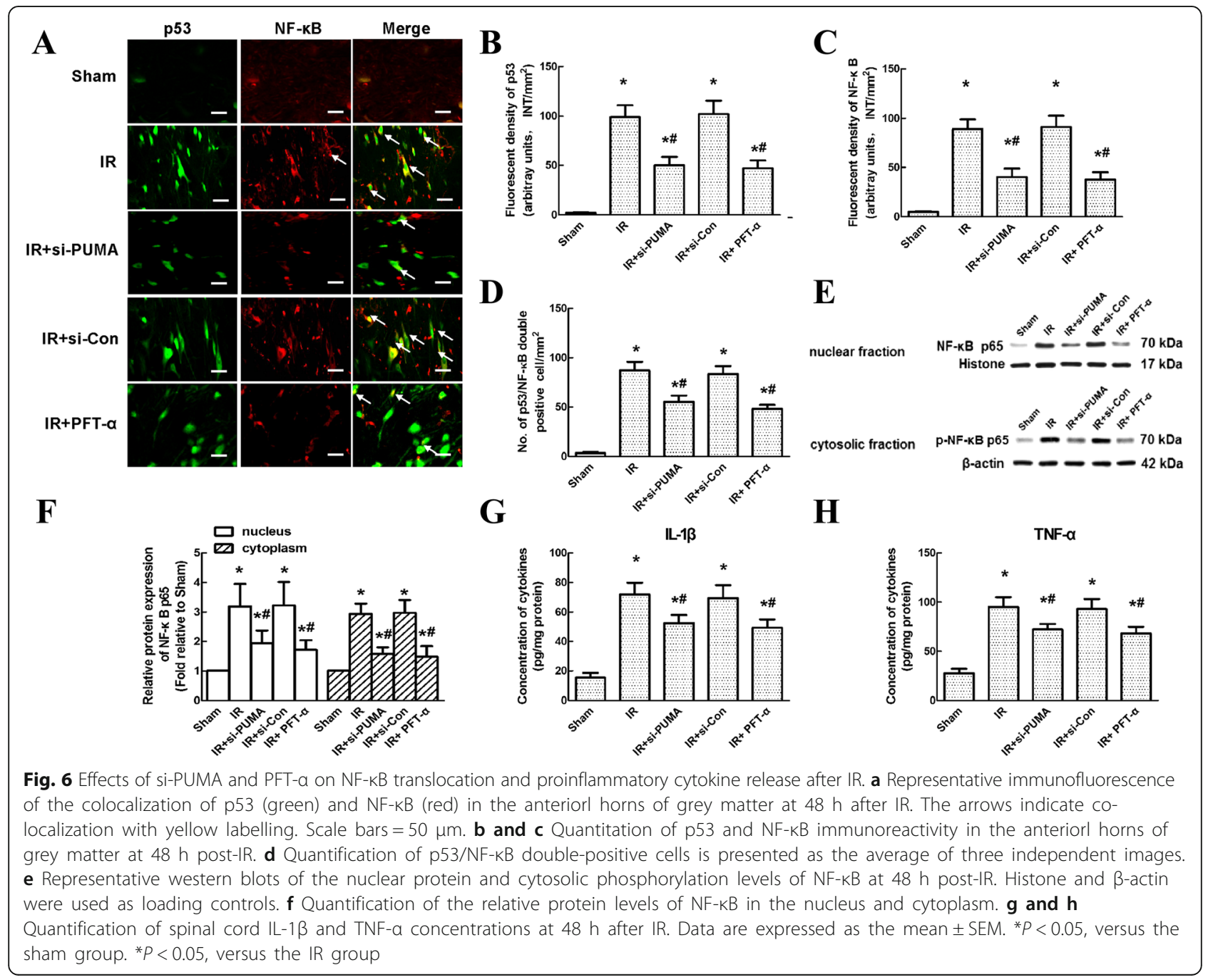


Intrathecal injection of si-PUMA and PFT-a preserved BSCB integrity after IR

EB extravasation was visualized as red spots under a fluorescence microscope. The representative images in Fig. 7a show that almost no red fluorescent spots were observed in the sham group. Compared with that in the sham group, a widespread distribution of red fluorescent spots that were much more intense was detected in the IR group. Injection of si-PUMA and PFT- $\alpha$ significantly decreased the number and intensity of the red spots. No differences were detected between the IR and si-Con groups $(P>0.05)$. Quantitation of the EB content and fluorescence intensity confirmed the above results (Fig. $7 \mathrm{~b}$ and $\mathrm{c}, P<0.05$ ).

Meanwhile, IR-induced increases in water content are greatly attributed to BSCB breakdown [21]. The spinal cord water content was also changed in a manner similar to that of EB extravasation (Fig. $7 \mathrm{~d}, P<0.05$ ).

\section{Discussion}

The important and diverse roles of p53 and its driven targets under stimuli have been widely explored in previous studies [6-8]. Under pathological conditions, several p53-mediated pathways are quickly activated and further funnelled into the common node of the p53 network via MDM2 to balance the survival or death of cells $[7,25]$. We demonstrated for the first time that PUMA, a target directly by p53 driven, also play crucial roles in spinal cord IR in addition to MDM2. Comprising the feedback loop, PUMA expression regulates the transactivation function of p53, which may further affect caspase 3-mediated apoptosis $[15,17,26]$. As expected, intrathecal treatment with si-PUMA and PFT- $\alpha$ exerted similar effects on preventing increased p53 and caspase 3 protein expressions. Additionally, the broader role of p53 in integrating with inflammatory pathways via NF- $\mathrm{kB}$ transcription has been reported [11]. Consistent with our study, comparable anti-inflammatory effects were also observed in rats injected with si-PUMA and PFT- $\alpha$, as similar preservations of BSCB integrity and decreased protein levels of NF- $\mathrm{kB}$ and cytokines IL-1 $\beta$ and TNF- $\alpha$ were observed. Together, these results suggested that the p53-PUMA feedback loop functions as a common node to regulate cellular apoptosis and neuroinflammation during spinal cord IR.

Apoptosis is now known to be the most important factor related to neuronal loss during IR [22]. PUMA, a wellknown p53-induced BH3-only protein, has been well demonstrated to regulate the apoptosis-promoting activity of tumour cells $[27,28]$. PUMA is expressed at a very low level under normal conditions, and upregulating PUMA expression using the adenoviral gene delivery technique in ovarian cancer cells is thought to exert better apoptosis-inducing effects but lower proliferation-inhibiting effects than those in cells transfected with the p53 gene [27]. Simultaneously, PUMA is closely related to p53 activity, as they

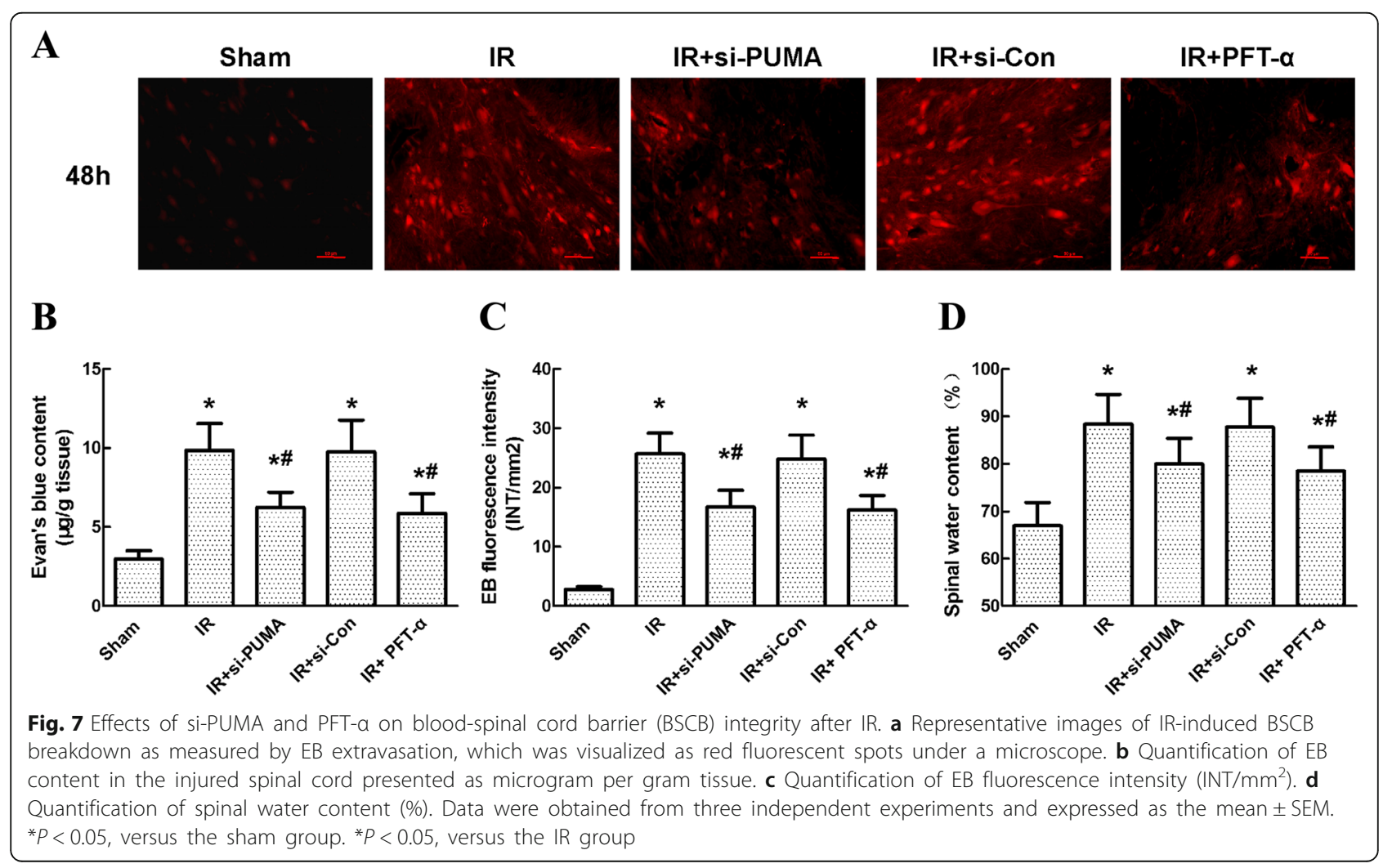


compose the feedback loop [16, 17]. PUMA expression is induced immediately after p53 translocation to the nucleus and consequently activates the execution of neuronal and myocardial apoptosis via the caspase cascade [15, 28, 29]. Silencing PUMA expression in cultured neurons prevented apoptosis to an extent similar to that in p53-deficient neurons [30]. A possible explanation for this phenomenon might be the decreased ability of PUMA to interact with p53, which, in turn, prevents p53 translocation and its transactivation ability to trigger cytochrome $\mathrm{c}$ release and activate caspase $3[16,17,28]$. In an in vitro study exploring the binding properties of p53 with PUMA and NOXA using a GST pull-down assay, the his-tagged p53 fusion protein was pulled down effectively with the BH3s of GST-PUMA and GST-NOXA, indicating that both the PUMA and NOXA BH3 regions interact with p53 in vitro [28]. Moreover, using the modelled complex structures, the predicted interaction sites further confirmed the structural interaction between p53 and PUMA [28]. Thus, asserting PUMA as a new treatment target in the regulation of cellular apoptosis during spinal cord IR is reasonable. Consistent with our study, PUMA and p53 protein are expressed at very low levels in normal spinal cord tissues. They are similarly rapidly increased with time over the same reperfusion period. To further clarify their interactions, we blocked PUMA and p53 functions by intrathecally injecting si-PUMA and PFT- $\alpha$ when their expression levels peaked. The decreased PUMA protein expression was changed consistently with the decreased p53 level. No significant differences were observed between treatment with si-PUMA and PFT- $\alpha$ (Fig. 4). In addition, some studies found that PUMA localized in mitochondria can decrease the ratio of the anti-apoptotic $\mathrm{Bcl}-2$ protein to the pro-apoptotic Bax protein and then induce caspase activation $[15,30]$. Thus, we also detected the protein levels of PUMA and p53 in subcellular fractions in this study. As shown by western blot analysis at $48 \mathrm{~h}$ post-IR, the protein levels of PUMA in the mitochondrial fraction were substantially increased at $48 \mathrm{~h}$ post-IR. In line with previous observations, the translocation of p53 to the nucleus indicated p53 transactivation $[13,15,30]$. As mutual factors in the feedback loop, the nuclear level of p53 was simultaneously significantly increased. Not surprisingly, downregulating PUMA via si-PUMA prevented increased mitochondrial PUMA and nuclear p53 levels to an extent similar to that achieved using the p53-specific inhibitor PFT- $\alpha$. These results confirmed the feedback regulatory effects on PUMA and p53 during spinal cord IR and that PUMA directly regulates the p53-PUMA feedback loop.

p53 and its driven proteins are clearly activated in a tissue or cell type-specific manner, thus accounting for the variability in the diverse mechanisms regulated by p53 and the proteins it drives [25, 30]. In neurons, IR-induced damage can overwhelm repair systems and initiate caspase-mediated neuronal apoptosis [14, 22]. As shown herein, upregulated p53 and PUMA fluorescent labels colocalized with neuronal labels at $48 \mathrm{~h}$ post-IR (Fig. 3). Having many typical and common characteristics of currently known caspases, caspase 3 plays a crucial role in the execution phase of cellular apoptosis [22]. To further determine whether the p53-PUMA loop regulates neuronal apoptosis, TUNEL staining and cytosolic caspase 3 levels were examined simultaneously. A significant level of apoptosis was induced as the levels of PUMA and p53 increased, demonstrated as decreased Tarlov scores, but the number of TUNEL-positive cells and cleaved caspase 3 protein levels increased. Consistent with a previous in vivo study, transgenic mice carrying a PUMA mutation were protected against an apoptotic stimulus similarly to p53deficient mice [31]. In the present study, intrathecal treatment with si-PUMA and PFT- $\alpha$ exerted similar effects on maintaining Tarlov scores and preventing increases in TUNEL-positive cells and cleaved caspase 3 levels in the spinal cord. In accordance with the abovementioned biochemical changes, our morphological evaluation by DAPI staining further confirmed that si-PUMA and PFT- $\alpha$ treatment similarly reduced the number of nuclei with typically apoptotic morphology, underscoring the decreased ability of apoptosis due to inhibition of the PUMA-p53 feedback loop.

Neuroinflammation is another major crucial factor contributing to the pathophysiological mechanisms of IR $[18,21,22]$. Emerging evidence suggests broader roles of p53 in interacting with the inflammatory pathway by modulating glial function and promoting NF- $\mathrm{KB}$ transcription [11, 32, 33]. For example, microglial secretion of the inflammatory cytokines IL- $1 \beta$ and TNF- $\alpha$ was demonstrated to be p53-dependent in neurodegenerative diseases, whereas the inhibition of p53 by PFT- $\alpha$ reversed the above proinflammatory phenotypic change in microglial cells [32]. Consistent with this observation, our double immunofluorescence staining confirmed that increased p53 and PUMA fluorescent labels were also widely distributed in activated astrocytes (displayed as hypertrophic body size) and microglial cells (displayed as amoeboid morphology). Moreover, the increased fluorescent p53 labels coincidentally localized with the NF- $k B$ labels, indicating their potential functional crosstalk in IR-induced inflammatory responses (Fig. 6). In the $\mathrm{NF}-\mathrm{kB}$ signalling cascade, phosphorylation is the essential step for the translocation of NF- $\mathrm{KB}$ from the cytoplasm to the nucleus, which then activates downstream transcription [24]. Thus, the nuclear localization and/or the use of phospho-specific antibodies to detect phosphorylation levels are two common methods for detecting NF- $\mathrm{KB}$ activation. Consistent with our previous studies in vivo, the increased nuclear translocation of NF- $\mathrm{kB}$ and glial production of cytokines are the quintessential markers of NF-kB-mediated neuroinflammation 
in injured spinal cords [34]. Recently, one study suggested that the different phosphorylation sites of the p65 sub-unit result in different levels of NF-kB transcriptional activity. Neither phosphorylation in cytoplasm nor translocation to nucleus alone is not sufficient to indicate NF-kB transcription. It was important to assess both, especially in the absence of an NF- $\kappa B$ transcription-dependent functional read out [24]. Thus, we assessed both indexes in this study. As shown in Fig. 6, the number of p53/NF- $\mathrm{kB}$ double-labelled cells, the nuclear protein and cytosolic phosphorylation levels of NF- $\mathrm{KB}$ and the accompanying levels of IL- $1 \beta$ and TNF- $\alpha$ were all greatly inhibited by treatment with si-PUMA and PFT- $\alpha$. Additionally, some published articles have emphasized that BSCB integrity is another useful factor for evaluating neuroinflammation after IR [18, 21]. Increased BSCB leakage can easily be recognized by substantial increases in spinal water content and EB extravasation [21]. As expected, substantially improved BSCB integrity was achieved by controlling the cytokine content by treatment with si-PUMA and PFT- $\alpha$. These findings provided clear evidence of the effects of the p53-PUMA feedback loop on neuroinflammation after IR.

Notably, contradictory p53 expression has been observed in astrocytes [14, 35]. For example, p53 was not obviously detectable in activated astrocytes at $24 \mathrm{~h}$ or $72 \mathrm{~h}$ after ischaemia but was clearly induced at 7 days post-injury [14]. These discrepancies were most likely attributed to the use of different disease models and the time points utilized to study specific glial cell activation. In addition, sequential activation and crosstalk between microglia and astrocytes are common in vivo, but they do not always occur in the same order given the diverse mechanisms of diseases [36]. Additionally, neuron-glial signalling exists not only at synaptic and axonal contacts but also via direct interaction with neuronal cell bodies [37]. In this context, the complexity of the neuron-glia architecture in the mammalian CNS greatly hindered the detailed determination of the neuron-glia pathophysiological functions and interactions in vivo. Therefore, in this study, we only confirmed the cellular location of p53 and PUMA in spinal cells and explored the net effects of si-PUMA treatment in rats by detecting the BSCB integrity and hind-limb motor function, regardless of how si-PUMA was regulated among spinal cells. Further in vitro studies are needed to investigate the mechanism underlying the p53-PUMA feedback loop in regulating other mediators to better elucidate its common node in multiple IR mechanisms.

\section{Conclusions}

In conclusion, this study explored the roles of p53 and its driven target PUMA in spinal cord IR. Inhibition of aberrant p53-PUMA feedback loop activation by the intrathecal injection of si-PUMA and PFT- $\alpha$ prevented IR-induced apoptosis, inflammatory responses and BSCB breakdown by inhibiting the activation of caspase 3-mediated apoptosis and NF-kB-mediated cytokine release. These results suggest potential new strategies for IR treatment.

\section{Abbreviations}

BCl-2: B-cell lymphoma-2; Bid: BH3-interacting domain death agonist; BSCB: Blood-spinal cord barrier; DAPI: 4',6-diamidino-2-phenylindole; DMSO: Dimethyl sulfoxide; IL: Interleukin; IR: Ischemia reperfusion; MDM2: Murine double minute 2 gene; NF: Nuclear factor; PBS: Phosphatebuffered saline; PUMA: p53 upregulated modulator of apoptosis; TNF: Tumor necrosis factor; TUNEL: Terminal deoxynucleotidyl-transferase mediated dUTP nick-end labelling

\section{Funding}

This work was supported by the National Natural Science Foundation of China (No. 81601053), the Scientific Research Project of Liaoning Province Department of Education (No. LK201636) and the Science and Technology Program of Liaoning (No. 2012408002).

\section{Availability of data and materials}

The materials supporting the conclusions of this article are all included within the article.

\section{Authors' contributions}

X-QL, F-SC and QY participated in the animal care and generated the animal models. QY and W-FT prepared the spinal cord tissues and performed the RT-PCR, ELISA and immunohistochemistry assays. F-SC, W-FT and Z-ZL performed the western blotting and statistical analyses. X-QL and QY wrote the manuscript. HM guided the study design and provided important suggestions on the manuscript preparation. All authors read and approved the final manuscript.

\section{Ethics approval}

All experimental protocols were approved by the Ethics Committee of China Medical University and performed in accordance with the Guide for the Care and Use of Laboratory Animals (U.S. National Institutes of Health publication no. 85-23, National Academy Press, Washington, DC, USA).

\section{Consent for publication}

Not applicable.

\section{Competing interests}

The authors declare that they have no competing interest.

\section{Publisher's Note}

Springer Nature remains neutral with regard to jurisdictional claims in published maps and institutional affiliations.

\section{Author details}

'Department of Anesthesiology, First Affiliated Hospital, China Medical University, Shenyang 110001, Liaoning, China. ${ }^{2}$ Department of Thoracic Surgery, Fourth Affiliated Hospital, China Medical University, Shenyang 110032, Liaoning, China.

Received: 7 May 2018 Accepted: 5 August 2018

Published online: 01 September 2018

\section{References}

1. Eltzschig HK, Eckle T. Ischemia and reperfusion--from mechanism to translation. Nat Med. 2011;17(11):1391-401.

2. $\mathrm{Hu}$ J, Yu Q, Xie L, Zhu H. Targeting the blood-spinal cord barrier: a therapeutic approach to spinal cord protection against ischemia-reperfusion injury. Life Sci. 2016;158:1-6.

3. Fu J, Sun $H$, Zhang $Y, X u$ W, Wang $C$, Fang $Y$, et al. Neuroprotective effects of luteolin against spinal cord ischemia-reperfusion injury by attenuation of oxidative stress, inflammation, and apoptosis. J Med Food. 2018;21 (1):13-20. 
4. Winklewski PJ, Radkowski M, Demkow U. Cross-talk between the inflammatory response, sympathetic activation and pulmonary infection in the ischemic stroke. J Neuroinflammation. 2014;11:213.

5. Parker BL, Shepherd NE, Trefely S, Hoffman NJ, White MY, Engholm-Keller K, et al. Structural basis for phosphorylation and lysine acetylation cross-talk in a kinase motif associated with myocardial ischemia and cardioprotection. $J$ Biol Chem. 2014;289(37):25890-906.

6. Liao JM, Cao B, Zhou X, Lu H. New insights into p53 functions through its target microRNAs. J Mol Cell Biol. 2014;6(3):206-13.

7. Vecino R, Burguete MC, Jover-Mengual T, Agulla J, Bobo-Jiménez V, Salom $J B$, et al. The MDM2-p53 pathway is involved in preconditioning-induced neuronal tolerance to ischemia. Sci Rep. 2018;8(1):1610.

8. Garufi A, Pistritto G, Baldari S, Toietta G, Cirone M, D'Orazi G. p53-dependent PUMA to DRAM antagonistic interplay as a key molecular switch in cellfate decision in normal/high glucose conditions. J Exp Clin Cancer Res. 2017;36(1):126.

9. Tu YF, Jiang ST, Chow YH, Huang CC, Ho CJ, Chou YP. Insulin receptor substrate-1 activation mediated p53 downregulation protects against hypoxic-ischemia in the neonatal brain. Mol Neurobiol. 2016;53(6):3658-69.

10. Lu H, Wang B. SIRT1 exerts neuroprotective effects by attenuating cerebral ischemia/reperfusion-induced injury via targeting p53/microRNA-22. Int J Mol Med. 2017;39(1):208-16.

11. Muñoz-Fontela C, Mandinova A, Aaronson SA, Lee SW. Emerging roles of p53 and other tumour-suppressor genes in immune regulation. Nat Rev Immunol. 2016;16(12):741-50

12. Cooks T, Harris CC, Oren M. Caught in the cross fire: p53 in inflammation. Carcinogenesis. 2014;35(8):1680-90.

13. Hong LZ, Zhao XY, Zhang HL. p53-mediated neuronal cell death in ischemic brain injury. Neurosci Bull. 2010;26(3):232-40.

14. Huang YN, Yang LY, Greig NH, Wang YC, Lai CC, Wang JY. Neuroprotective effects of pifithrin-a against traumatic brain injury in the striatum through suppression of neuroinflammation, oxidative stress, autophagy, and apoptosis. Sci Rep. 2018;8(1):2368

15. Niizuma K, Endo H, Nito C, Myer DJ, Chan PH. Potential role of PUMA in delayed death of hippocampal CA1 neurons after transient global cerebral ischemia. Stroke. 2009;40(2):618-25.

16. Tu S, Liu ZQ, Fu JJ, Zhu WF, Luo DY, Wan FS. Inhibitory effect of p53 upregulated modulator of apoptosis targeting siRNA on hypoxia/reoxygenation-induced cardiomyocyte apoptosis in rats. Cardiology. 2012;122(2):93-100.

17. Toth A, Jeffers JR, Nickson P, Min JY, Morgan JP, Zambetti GP, et al. Targeted deletion of PUMA attenuates cardiomyocyte death and improves cardiac function during ischemia-reperfusion. Am J Physiol Heart Circ Physiol. 2006;291(1):H52-60.

18. Li XQ, Chen FS, Tan WF, Zhang ZL, Fang B, Ma H. Elevated microRNA-129$5 p$ level ameliorates neuroinflammation and blood-spinal cord barrier damage after ischemia-reperfusion by inhibiting HMGB1 and the TLR3cytokine pathway. J Neuroinflammation. 2017;14:205.

19. Tarlov IM. Acute spinal cord compression paralysis. J Neurosurg. 1972; 36(1):10-20

20. Gong G, Yuan LB, Hu L, Wu W, Yin L, Hou JL, Liu YH, Zhou LS. Glycyrrhizin attenuates rat ischemic spinal cord injury by suppressing inflammatory cytokines and HMGB1. Acta Pharmacol Sin. 2012;33(1):11-8.

21. Fang $B$, Li XM, Sun XJ, Bao NR, Ren XY, Lv HW, Ma H. Ischemic preconditioning protects against spinal cord ischemia-reperfusion injury in rabbits by attenuating blood spinal cord barrier disruption. Int J Mol Sci. 2013;14(5):10343-54.

22. Li XQ, Cao XZ, Wang J, Fang B, Tan WF, Ma H. Sevoflurane preconditioning ameliorates neuronal deficits by inhibiting microglial MMP-9 expression after spinal cord ischemia/reperfusion in rats. Mol Brain. 2014;7:69.

23. Jeong SJ, Radonovich M, Brady JN, Pise-Masison CA. HTLV-I tax induces a novel interaction between p65/RelA and p53 that results in inhibition of p53 transcriptional activity. Blood. 2004;104(5):1490-7.

24. Maguire O, O'Loughlin K, Minderman H. Simultaneous assessment of NF-kB/ p65 phosphorylation and nuclear localization using imaging flow cytometry. J Immunol Methods. 2015;423:3-11.

25. Hu W, Feng Z, Levine AJ. The regulation of multiple p53 stress responses is mediated through MDM2. Genes Cancer. 2012;3(3-4):199-208.

26. Tichy A, Marek J, Havelek R, Pejchal J, Seifrtova M, Zarybnicka L, et al. New light on an old friend: targeting PUMA in radioprotection and therapy of cardiovascular and neurodegenerative diseases. Curr Drug Targets. 2018;19. https://doi.org/10.2174/1389450119666180406110743
27. Luan QC, Sun YR, Han P, Chen Y. Ad-PUMA sensitizes ovarian cancer cells to chemotherapeutic agents. Eur Rev Med Pharmacol Sci. 2015;19(23):4525-32.

28. Park SY, Jeong MS, Jang SB. In vitro binding properties of tumor suppressor p53 with PUMA and NOXA. Biochem Biophys Res Commun. 2012;420(2):350-6.

29. Chen $H$, Tian $M$, Jin $L$, Jia $H$, Jin $Y$. PUMA is involved in ischemia/ reperfusion-induced apoptosis of mouse cerebral astrocytes. Neuroscience. 2015;284:824-32. https://doi.org/10.1016/j.neuroscience.2014.10.059.

30. Cregan SP, Arbour NA, MacLaurin JG, Callaghan SM, Fortin A, Cheung ECC et al. p53 activation domain 1 is essential for PUMA upregulation and p53-mediated neuronal cell death. J Neurosci. 2004;24:10003-12.

31. Villunger A, Michalak EM, Coultas L, Müllauer F, Böck G, Ausserlechner MJ, et al. p53- and drug-induced apoptotic responses mediated by BH3-only proteins puma and noxa. Science. 2003;302(5647):1036-8.

32. Jebelli J, Hooper C, Pocock JM. Microglial p53 activation is detrimental to neuronal synapses during activation-induced inflammation: implications for neurodegeneration. Neurosci Lett. 2014;583:92-7.

33. Turnquist C, Horikawa I, Foran E, Major EO, Vojtesek B, Lane DP, et al. p53 isoforms regulate astrocyte-mediated neuroprotection and neurodegeneration. Cell Death Differ. 2016;23(9):1515-28.

34. Li XQ, Wang J, Fang B, Tan WF, Ma H. Intrathecal antagonism of microglial TLR4 reduces inflammatory damage to blood-spinal cord barrier following ischemia/reperfusion injury in rats. Mol Brain. 2014;7:28. d

35. Chung YH, Shin CM, Kim MJ, Lee EY, Kim G, Cha Cl. Enhanced expression of p53 in reactive astrocytes following transient focal ischemia. Neurol Res. 2002;24(3):324-8.

36. Norden DM, Trojanowski PJ, Villanueva E, Navarro E, Godbout JP. Sequential activation of microglia and astrocyte cytokine expression precedes increased Iba-1 or GFAP immunoreactivity following systemic immune challenge. Glia. 2016;64(2):300-16.

37. Coutinho-Budd JC, Sheehan AE, Freeman MR. The secreted neurotrophin Spätzle 3 promotes glial morphogenesis and supports neuronal survival and function. Genes Dev. 2017;31(20):2023-38.

\section{Ready to submit your research? Choose BMC and benefit from:}

- fast, convenient online submission

- thorough peer review by experienced researchers in your field

- rapid publication on acceptance

- support for research data, including large and complex data types

- gold Open Access which fosters wider collaboration and increased citations

- maximum visibility for your research: over $100 \mathrm{M}$ website views per year

At BMC, research is always in progress.

Learn more biomedcentral.com/submissions 